\title{
Exercise medicine for prostate cancer
}

\author{
Robert U. Newton • Daniel A. Galvão
}

Received: 4 December 2012 / Accepted: 8 December 2012 /Published online: 11 January 2013

(C) European Group for Research into Elderly and Physical Activity (EGREPA) 2013

\begin{abstract}
Since initial reports in the mid-1980s, there has been increasing interest in the application of exercise as medicine for the prevention and management of cancer. A large number of high-quality, randomised, controlled trials with cancer survivors have confirmed both aerobic and resistance exercise to be highly beneficial for improving body composition, quality of life, mental health functional capacity and reducing risk of cancer recurrence and development of other chronic diseases. Such benefits have ultimately been realised in reduced cancer mortality between 30 and $60 \%$ in large cohort retrospective studies. Treatments for prostate cancer are increasingly effective with quite high 5- and 10-year survival rates; however, side effects of endocrine treatments in particular impact on quality of life and increased co-morbidities for the survivor. Testosterone deprivation while highly effective for controlling prostate cancer growth results in loss of muscle and bone, increased fat mass, increased incidence of metabolic syndrome, cardiovascular disease and sudden death. Exercise has been demonstrated to be a very effective medicine for counteracting all of these treatment toxicities as well as improving mental health and quality of life. Exercise has been demonstrated to be safe and well tolerated by cancer patients. Current recommendation is to complete at least $150 \mathrm{~min}$ of aerobic exercise and two or more sessions of resistance training per week. More specific exercise prescription is required to address particular treatment toxicities such as bone loss or obesity. This paper is a review of key research from our group into exercise medicine for prostate cancer.
\end{abstract}

R. U. Newton $(\bowtie) \cdot$ D. A. Galvão

Edith Cowan University Health and Wellness Institute,

Edith Cowan University, 270 Joondalup Drive,

Joondalup, Western Australia 6027, Australia

e-mail: r.newton@ecu.edu.au

D. A. Galvão

e-mail:d.galvao@ecu.edu.au
Keywords Prostate cancer - Androgen deprivation therapy · Exercise $\cdot$ Resistance training $\cdot$ Aerobic training $\cdot$ Side effects

\section{Background}

The earliest publication espousing the benefits of physical exercise for cancer patients was published by Winningham et al. in 1986 in The Physician and Sports Medicine [25]. To quote this landmark paper, "clinical observations indicate that exercise is [a] promising restorative technique for cancer patients but it is a fairly new concept." Up until this time and in fact, for the next 15 years or so, clinicians advised cancer patients to rest and avoid activity so that they could better tolerate the rigours of undergoing treatment [21]. However, we now have 20 years of research demonstrating that exercise plays a pivotal role in cancer prevention and management [21]. In 2005, we published a review of all exercise intervention studies in cancer patients up until June 2004 [5]. At this time, there were 26 trials with the majority of studies in breast cancer and utilising cardiovascular or aerobic exercise as the modality. Despite the fact that many of these studies implemented exercise of interventions of relatively short duration, suboptimal exercise selection and relatively low intensities on the threshold for driving training adaptation, the benefits were quite considerable and consistent. There were no reported adverse events directly attributable to the exercise interventions. The major benefits reported were reduced fatigue, improved muscle function and physical performance, increased aerobic capacity, enhanced body composition and improved quality of life [5].

Worldwide, prostate cancer is the most common cancer in men (apart from non-melanoma skin cancers) and the second most common cause of cancer death in men [23]. In Australia, incidence of prostate cancer is on the increase not only because of the ageing demography of the world population but also because of the introduction of the prostatespecific antigen (PSA) test providing more frequent and earlier detection [14]. Although still somewhat speculative, 
the reduction in habitual physical activity in men may also be contributing to increased incidence of prostate cancer [4]. Fortunately, mortality rates for prostate cancer are actually decreasing due to early detection and improve treatments [14]. The common treatments for prostate cancer include active surveillance (non-treatment), surgical removal of the prostate, radiation therapy by either external beam or brachytherapy, androgen deprivation therapy (ADT) and chemotherapy for metastatic disease and for patients that no longer respond to ADT [17].

Androgen deprivation is achieved through either or both elimination of testosterone production to administration of luteinising hormone releasing hormone antagonists or by drugs which block the androgen receptors preventing testosterone from signalling to the cells. ADT is being increasingly used in the management of prostate cancer, and while highly effective for slowing prostate cancer growth, it does result in a range of toxicities which impact negatively on the man and to varying degrees [3].

We have previously reported [8] clinically significant decreases in the upper limb, lower limb, trunk and whole body lean mass of 5.6, 3.7, 1.4 and $2.4 \%$, respectively, while fat mass increased by 20.7, 18.7, 12.0 and 13.8 over the course of 36 weeks of ADT. Further, hip, spine, whole body and upper limb bone mineral density (BMD) decreased by 1.9, 3.3, 1.6 and $1.3 \%$, respectively, but not lower limb BMD during this period. In addition, physical activity levels decreased, and levels of fatigue increased. We have also recently reported no recovery to pre-treatment levels of lean and fat mass after 2 years of follow-up with this cohort. Patients who failed to recover testosterone by 2 years post-treatment experienced significant further increases in fat mass compared with those who recovered eugonadal levels of testosterone [24]. In a subsequent cross-sectional study [12] comparing men on long-term ADT with matched controls without prostate cancer, we reported that men on ADT had significantly reduced muscle strength for the upper and lower body and impaired functional performance compared to controls. As expected, ADT patients had significantly lower whole body and hip BMD and higher per cent of body fat than controls and tended to have lower whole body lean mass $(-2.3 \mathrm{~kg})$. Men undertaking ADT were consistently impaired across a broad range of physical and functional musculoskeletal performance assessments compared with their age-matched normal controls.

These ADT toxicities create a "perfect storm" for falls, fracture and the poor prognosis resulting from such sequelae in these men as they are predominantly of old age. ADT compromises balance and motor control both centrally through deprivation of testosterone to the brain and reduced ability of the motor system to respond effectively to a slip or trip. Muscle size, strength and power are reduced, reducing the ability to recover from loss of balance. Prevalence of osteopenia and osteoporosis increase with the number of years on ADT such that by 10 years, $100 \%$ of patients will have lower than normal BMD [18]. The combination of low bone density, compromised balance and ability to functionally respond causes increased risk of fracture of $50 \%$ or more [1].

The other arrays of ADT toxicities encompass metabolic syndrome, type II diabetes and the increased risk of cardiovascular disease [3]. For example, it has been reported [15] that $\mathrm{ADT}$ is associated with an increased risk of diabetes (44\%), coronary heart disease (16\%), myocardial infarction (11\%) and sudden death (16\%), causing patients and clinicians to question the efficacy of ADT and possibly declining treatment. This is not without justification, as the majority of men with prostate cancer will die of diseases other than their primary malignancy [3]. These treatment toxicities are of considerable concern and lead our team to embark on a series of studies to evaluate the efficacy of physical exercise to prevent or reverse such side effects.

Our first study [7] involved 11 men receiving ADT for their prostate cancer. All participants completed a 20-week resistance training program consisting of two sessions per week of 12 exercises in the intensity range of 6 to 12 repetitions maximum (RM). This corresponds to a weight that can only be lifted 6 to 12 times. Muscle strength (chest press, $40 \%$; seated row, $42 \%$; leg press, $96 \%$ ) and muscle endurance (chest press, $115 \%$; leg press, $167 \%$ ) increased significantly after training. We also reported significant improvement in the 6-m usual walk (14.1\%), 6-m backwards walk $(22.3 \%)$, chair rise $(26.8 \%)$, stair climbing (10.4\%), 400-m walk (7.4\%) and balance (7.8\%). Muscle thickness increased by $15.7 \%$ at the quadriceps site. Whole body lean mass was preserved with no change in fat mass. There were no significant changes in PSA, testosterone, GH, cortisol or haemoglobin. We concluded that progressive resistance exercise has beneficial effects on muscle strength, functional performance and balance in older men receiving androgen deprivation for prostate cancer and should be considered to preserve body composition and reduce treatment side effects. This was an important early study of resistance training in this population because we demonstrated, first, that men without testosterone could respond to an appropriate exercise program and, second, that the 20 -week resistance training intervention period did not compromise therapy intent, that is, there was no rise in PSA or testosterone. In a follow-up study, we reported that testosterone remains suppressed even immediately following an acute bout of high intensity resistance exercise [6].

The next study [10] was a randomized controlled trial with 57 patients with prostate cancer undergoing AST (commenced $>2$ months prior) randomly assigned to a program of resistance and aerobic exercise $(n=29)$ or usual care $(n=28)$ for 12 weeks. Patients undergoing exercise showed an increase in lean mass compared with usual care $(0.8 \mathrm{~kg})$ and similarly better muscle strength (leg press, $31 \mathrm{~kg}$ ) and 6-m 
walk time $(0.31 \mathrm{~s})$. Exercise also improved several aspects of quality of life including general health, reduced fatigue and decreased levels of C-reactive protein. There were no adverse events during the testing or exercise intervention program. Our conclusion was that relatively brief exposure to exercise significantly improved muscle mass, strength, physical function and balance in hypogonadal men compared with normal care. The exercise regimen was well tolerated and could be recommended for patients undergoing $\mathrm{ADT}$ as an effective countermeasure to these common treatment-related adverse effects.

It was perplexing that a 12 -week exercise intervention resulted in no significant reduction in body fat. To investigate this further, we separated and compared those patients who were acutely ( $<6$ months) or chronically ( $>6$ months) experiencing ADT [11]. Patients on acute androgen deprivation showed an increase in total body fat compared to those on chronic ADT $(0.9 \mathrm{~kg})$. Each group experienced increased appendicular skeletal muscle (about $0.5 \mathrm{~kg}$ ). Triglycerides decreased in the chronic group and increased in the acute group. Change in triglycerides was associated with the change in total body fat $(r=0.411)$. There were no differences between the groups in PSA, testosterone, glucose, insulin, total cholesterol, low- and high-density lipoproteins, cholesterol, C-reactive protein, homocysteine or quality of life. The two groups showed similar improvement in muscle strength and function, and cardiovascular fitness as a result of the exercise intervention. We concluded that apart from differences in body fat and triglycerides, the beneficial effects of exercise are similar in patients on acute or chronic androgen suppression therapy.

It was time to embark on a much longer duration clinical trial of exercise, in particular to address bone outcomes, which require a minimum of 6 months intervention to realize any benefit. This study [19] was 12 months in duration with the specific purpose of examining the effects of long-term exercise on reversing musculoskeletal-related side effects and cardiovascular and diabetes risk factors in men receiving androgen deprivation for their prostate cancer. Specifically, we aimed to investigate the effects of a 12-month exercise program designed to load the musculoskeletal system and reduce cardiovascular and diabetes disease progression on the following primary endpoints: (1) bone mineral density, (2) cardiorespiratory function and maximal oxygen capacity, (3) body composition (lean mass and fat mass), (4) blood pressure and cardiovascular function, (5) lipids and glycaemic control and (6) quality of life and psychological distress. We implemented a multi-site, randomized, controlled trial of 195 men (65 subjects per arm) undergoing treatment for prostate cancer involving ADT. Participants were randomized to (1) resistance/impact loading exercise, (2) resistance/cardiovascular exercise groups and (3) usual care/delayed exercise. Participants then completed progressive training for 12 months.
The impact exercise was particularly unique as previous research had demonstrated that skipping, bounding and depth jumping exercises were effective for reversing bone loss in menopausal women [2]. An early finding [20] was that usual care resulted in a $3.2 \%$ loss of lumbar BMD, and rather surprisingly, the group completing a combination of resistance and aerobic exercise also lost $2.6 \%$. Both of these changes were significant. The third group which completed a combination of resistance training and the impact loading protocol exhibited no significant change in bone mineral density over the same time period. Clearly, different modes of exercise effect different aspects of ADT toxicity and exercise description must be highly specific to address the individual problems faced by these patients. Another example of exercise specificity in this patient population is that we are currently exploring the feasibility of a modular exercise program for prostate cancer patients with bone metastases [9].

In 2009, our team published the Australian position stand on optimising cancer outcomes through exercise [13]. We reported that participating in exercise has also been associated with benefits during and following treatment for cancer, including improvements in psychosocial and physical outcomes, as well as better compliance with treatment regimens, reduced impact of disease symptoms and treatmentrelated side effects and survival benefits for particular cancers. The general exercise prescription for people undertaking or having completed cancer treatment was moderate intensity, regular frequency (3-5 times per week) for at least 20 min per session, involving aerobic, resistance or mixed exercise types. In 2010, the American College of Sports Medicine convened a roundtable of experts to formulate the exercise guidelines for cancer survivors [22]. This extensive review determined that there was overwhelming data from RCTs that exercise for prostate cancer survivors was safe, increased aerobic fitness and muscle strength and reduced fatigue. There was also evidence of improved body composition, quality of life and physical function.

While we have made the argument that exercise prescription must be specific to address the problems facing the patient (e.g. impact loading prescription for those with bone loss), the general recommendation is as follows [13]: Prostate cancer survivors should aim to accumulate at least $150 \mathrm{~min} /$ week of moderate aerobic exercise (between 6 and 8 on a ten-point perceived exertion scale) and equally important two or more sessions per week of resistance training involving three or more sets of six to eight exercises at an intensity of 6 to $10 \mathrm{RM}$.

The ultimate question is whether physical exercise can actually improve survival after prostate cancer diagnosis. The most significant paper today has demonstrated a $49 \%$ lower risk of all-cause mortality and a $61 \%$ lower risk of prostate cancer death in a follow-up of 2,705 men diagnosed with non-metastatic disease [16]. Certainly, it is critical to "avoid inactivity" even during difficult treatments. 


\section{Conclusions}

There is overwhelming evidence that appropriate exercise is safe and well tolerated by prostate cancer survivors and will result in improved aerobic fitness and muscle strength, as well as reduced fatigue. While further RCTs are required, current evidence suggests that exercise will also enhance quality of life and physical function, as well as maintain or improve healthy body composition. The large study by Kenfield et al. [16] has demonstrated that even a modest amount of vigorous exercise will result in $49 \%$ lower risk of all-cause mortality and $61 \%$ lower risk of prostate cancer death. However, the benefits of physical activity is highly specific to the exercise mode and dosage prescribed, and it appears that more sophisticated exercise programming is required in particular to address bone and fat mass outcomes.

Conflict of interest No competing interests.

Authors' contributions RUN and DAG researched and wrote the paper. Both authors contributed to and approved the final manuscript.

\section{References}

1. Ahlborg HG, Nguyen ND, Center JR, Eisman JA, Nguyen TV (2008) Incidence and risk factors for low trauma fractures in men with prostate cancer. Bone 43(3):556-560

2. Cheng S, Sipila S, Taaffe DR, Puolakka J, Suominen H (2002) Change in bone mass distribution induced by hormone replacement therapy and high-impact physical exercise in postmenopausal women. Bone 31(1):126-135

3. Collins L, Basaria S (2012) Adverse effects of androgen deprivation therapy in men with prostate cancer: a focus on metabolic and cardiovascular complications. Asian J Androl 14(2):222-225. doi:10.1038/aja.2011.109

4. Friedenreich CM, Neilson HK, Lynch BM (2010) State of the epidemiological evidence on physical activity and cancer prevention. Eur J Cancer 46(14):2593-2604. doi:10.1016/ j.ejca.2010.07.028

5. Galvão DA, Newton RU (2005) Review of exercise intervention studies in cancer patients. J Clin Oncol 23(4):899-909

6. Galvao DA, Nosaka K, Taaffe DR, Peake J, Spry N, Suzuki K, Yamaya K, McGuigan MR, Kristjanson LJ, Newton RU (2008) Endocrine and immune responses to resistance training in prostate cancer patients. Prostate Cancer Prostatic Dis 11(2):160-165. doi:10.1038/sj.pcan.4500991

7. Galvão DA, Nosaka K, Taaffe DR, Spry N, Kristjanson LJ, McGuigan MR, Suzuki K, Yamaya K, Newton RU (2006) Resistance training and reduction of treatment side effects in prostate cancer patients. Med Sci Sports Exer 38(12):20452052

8. Galvao DA, Spry NA, Taaffe DR, Newton RU, Stanley J, Shannon T, Rowling C, Prince R (2008) Changes in muscle, fat and bone mass after 36 weeks of maximal androgen blockade for prostate cancer. BJU Int 102(1):44-47. doi:10.1111/j.1464-410X.2008.07539.x

9. Galvao DA, Taaffe DR, Cormie P, Spry N, Chambers SK, PeddleMcIntyre C, Baker M, Denham J, Joseph D, Groom G, Newton RU (2011) Efficacy and safety of a modular multi-modal exercise program in prostate cancer patients with bone metastases: a randomized controlled trial. BMC Cancer 11:517. doi:10.1186/14712407-11-517

10. Galvao DA, Taaffe DR, Spry N, Joseph D, Newton RU (2010) Combined resistance and aerobic exercise program reverses muscle loss in men undergoing androgen suppression therapy for prostate cancer without bone metastases: a randomized controlled trial. J Clin Oncol 28(2):340-347. doi:10.1200/ JCO.2009.23.2488

11. Galvao DA, Taaffe DR, Spry N, Joseph D, Newton RU (2011) Acute versus chronic exposure to androgen suppression for prostate cancer: impact on the exercise response. J Urol 186(4):12911297. doi:10.1016/j.juro.2011.05.055

12. Galvão DA, Taaffe DR, Spry N, Joseph D, Turner D, Newton RU (2009) Reduced muscle strength and functional performance in men with prostate cancer undergoing androgen suppression: a comprehensive cross-sectional investigation. Prostate Cancer Prostatic Dis 12(2):198-203

13. Hayes SC, Spence RR, Galvao DA, Newton RU (2009) Australian Association for Exercise and Sport Science position stand: optimising cancer outcomes through exercise. J Sci Med Sport/Sports Med Aust 12(4):428-434. doi:10.1016/j.jsams.2009.03.002

14. Jemal A, Siegel R, Ward E, Hao Y, Xu J, Murray T, Thun MJ (2008) Cancer statistics, 2008. CA Cancer J Clin 58(2):71-96

15. Keating NL, O’Malley AJ, Smith MR (2006) Diabetes and cardiovascular disease during androgen deprivation therapy for prostate cancer. J Clin Oncol Off J Am Soc Clin Oncol 24(27):4448-4456

16. Kenfield SA, Stampfer MJ, Giovannucci E, Chan JM (2011) Physical activity and survival after prostate cancer diagnosis in the health professionals follow-up study. J Clin Oncol 29(6):726732. doi: $10.1200 / J C O .2010 .31 .5226$

17. Michaelson MD, Cotter SE, Gargollo PC, Zietman AL, Dahl DM, Smith MR (2008) Management of complications of prostate cancer treatment. CA Cancer J Clin 58(4):196-213. doi:10.3322/ CA.2008.0002

18. Morote J, Morin JP, Orsola A, Abascal JM, Salvador C, Trilla E, Raventos CX, Cecchini L, Encabo G, Reventos J (2007) Prevalence of osteoporosis during long-term androgen deprivation therapy in patients with prostate cancer. Urology 69 (3):500-504

19. Newton RU, Taaffe DR, Spry N, Gardiner RA, Levin G, Wall B, Joseph D, Chambers SK, Galvao DA (2009) A phase III clinical trial of exercise modalities on treatment side-effects in men receiving therapy for prostate cancer. BMC Cancer 9:210. doi:10.1186/ 1471-2407-9-210

20. Newton RU, Taaffe DR, Spry N, Joseph D, Cormie P, Gardiner R, Galvão DA (2011) High impact exercise preserves bone in men receiving ADT for prostate cancer. Paper presented at the 12th Australasian Prostate Cancer Conference, Melbourne, Australia, 3-5 August 2011

21. Schmitz KH, Courneya KS, Matthews C, Demark-Wahnefried W, Galvao DA, Pinto BM, Irwin ML, Wolin KY, Segal RJ, Lucia A, Schneider CM, von Gruenigen VE, Schwartz AL (2010) American College of Sports Medicine roundtable on exercise guidelines for cancer survivors. Med Sci Sports Exerc 42(7):1409-1426. doi:10.1249/MSS.0b013e3181e0c112

22. Schmitz KH, Courneya KS, Matthews C, Demark-Wahnefried W, Galvao DA, Pinto BM, Irwin ML, Wolin KY, Segal RJ, Lucia A, Schneider CM, von Gruenigen VE, Schwartz AL (2010) American College of Sports Medicine roundtable on exercise guidelines for 
cancer survivors. Med Sci Sports Exer 42(7):1409-1426. doi:10.1249/MSS.0b013e3181e0c112

23. Siegel R, DeSantis C, Virgo K, Stein K, Mariotto A, Smith T, Cooper D, Gansler T, Lerro C, Fedewa S, Lin C, Leach C, Cannady RS, Cho H, Scoppa S, Hachey M, Kirch R, Jemal A, Ward E (2012) Cancer treatment and survivorship statistics, 2012. CA Cancer J Clin 62(4):220-241. doi:10.3322/ caac. 21149
24. Spry NA, Taaffe DR, England PJ, Judge JS, Stephens DA, PeddleMcIntyre C, Baker MK, Newton RU, Galvao DA (2012) Longterm effects of intermittent androgen suppression therapy on lean and fat mass: a 33-month prospective study. Prostate Cancer Prostatic Dis. doi:10.1038/pcan.2012.33

25. Winningham ML, MacVicar MG, Burke CA (1986) Exercise for cancer patients: guidelines and precautions. Physician Sports Med 14(10):125-134 